\title{
Simulations of a classical spin system with competing superexchange and double-exchange interactions
}

\author{
Shan-Ho Tsai and D. P. Landau \\ Center for Simulational Physics, University of Georgia, Athens, GA 30602
}

\begin{abstract}
Monte-Carlo simulations and ground-state calculations have been used to map out the phase diagram of a system of classical spins, on a simple cubic lattice, where nearest-neighbor pairs of spins are coupled via competing antiferromagnetic superexchange and ferromagnetic double-exchange interactions. For a certain range of parameters, this model is relevant for some magnetic materials, such as doped manganites, which exhibit the remarkable colossal magnetoresistance effect. The phase diagram includes two regions in which the two sublattice magnetizations differ in magnitude. Spin-dynamics simulations have been used to compute the time- and space-displaced spin-spin correlation functions, and their Fourier transforms, which yield the dynamic structure factor $S(q, \omega)$ for this system. Effects of the double-exchange interaction on the dispersion curves are shown.
\end{abstract}

\section{INTRODUCTION}

Doped rare-earth manganites with general chemical formula $\mathrm{Re}_{1-x} \mathrm{~A}_{x} \mathrm{MnO}_{3}$ (where Re denotes a rare-earth and $\mathrm{A}$ is typically $\mathrm{Ca}$ or $\mathrm{Sr}$ ) have been shown to exhibit colossal magnetoresistance, and a wide range of other physical properties, which are strongly dependent upon doping [1]. The physical mechanisms responsible for the unusual magnetoresistance properties of these materials have not been understood yet, and it has been suggested that double-exchange interactions [2] between $\mathrm{Mn}^{+3}$ and $\mathrm{Mn}^{+4}$ ions are important. However, there is much ongoing debate as to whether double-exchange alone can indeed give rise to colossal magnetoresistance or if further mechanisms have to be considered 3 . It is thus important to further understand properties associated with the double-exchange theory.

Equal valence manganese ions in the parent compounds interact via a superexchange mechanism, whereas the different valence manganese ions, introduced through doping, are coupled via a double-exchange interaction [1, 2, 4]. We investigate a simple model of classical spins in which nearest-neighbor spins interact via a competing antiferromagnetic superexchange and a ferromagnetic double-exchange interaction, where the ratio of these two interactions mimic the effects of the doping $x$. Properties of the pure Heisenberg model (only superexchange interaction) are well-known [5] and recently a highaccuracy Monte-Carlo study obtained the paramagnetferromagnet transition temperature and associated static critical exponents of the pure double-exchange model [6]. Recent studies of the phase diagram of double-exchange systems using a different approach from ours are given in Ref. 7]. We use a combination of Monte-Carlo simulations and ground-state calculations to determine the phase diagram of the model with varying ratios of the superexchange and double-exchange interaction strengths. We also use spin-dynamics techniques to study the dynamic structure factor and obtain the dispersion curve of the double-exchange model.

\section{MODEL AND METHODS}

The model considered here can be described by the Hamiltonian

$$
\mathcal{H}=-J_{A F} \sum_{<\mathbf{r}, \mathbf{r}^{\prime}>} \mathbf{S}_{\mathbf{r}} \cdot \mathbf{S}_{\mathbf{r}^{\prime}}-J_{D E} \sum_{<\mathbf{r}, \mathbf{r}^{\prime}>} \sqrt{1+\mathbf{S}_{\mathbf{r}} \cdot \mathbf{S}_{\mathbf{r}^{\prime}}}
$$

where $\mathbf{S}_{\mathbf{r}}=\left(S_{\mathbf{r}}^{x}, S_{\mathbf{r}}^{y}, S_{\mathbf{r}}{ }^{z}\right)$ is a three-dimensional classical spin of unit length at site $\mathbf{r}$. The first term, with $J_{A F}<0$, is the antiferromagnetic superexchange interaction and the second term describes a ferromagnetic double-exchange term with coupling constant $J_{D E}>0$ between nearest-neighbor pairs of spins. We consider $L \times L \times L$ simple cubic lattices with periodic boundary conditions, and divide the system into two sublattices, $A$ and $B$, with magnetizations $\mathbf{m}_{A}$ and $\mathbf{m}_{B}$, respectively. The scalar product is defined as $\mathbf{m}_{A} \cdot \mathbf{m}_{B}=$ $\left|\mathbf{m}_{A}\right|\left|\mathbf{m}_{B}\right| \cos \theta \equiv y$, where clearly $-1 \leq y \leq 1$.

In the ground state, the condition of energy minimization leads to the expression

$$
y=\left(J_{D E}\right)^{2} /\left(4\left|J_{A F}\right|^{2}\right)-1
$$

with $\left|J_{A F}\right| / J_{D E} \geq 1 / \sqrt{8}=0.35355 \ldots$, and for $\left|J_{A F}\right| / J_{D E}<0.35355 \ldots$ the ground state is ferromagnetic. Eq.(2) admits the possibility of non-colinear phases and/or sublattice magnetizations with different magnitudes. Note that $y=0$ for $\left|J_{A F}\right| / J_{D E}=1 / 2$, which means that $\theta=\pi / 2$ and/or that the magnetization in at least one sublattice is zero in this case.

For $T>0$ we use Monte-Carlo simulations to measure thermodynamic quantities of the model in order to determine the phase diagram. We used the Metropolis algorithm, lattice sizes $L=12$ and 24, and typically we discarded 10000 Monte-Carlo steps for thermalization and used 10000-30000 steps in computing averages.

The dynamics of the spins are governed by the equations of motion

$$
\frac{d}{d t} \mathbf{S}_{\mathbf{r}}=\mathbf{S}_{\mathbf{r}} \times\left(-\vec{\nabla}_{\mathbf{r}} \mathcal{H}\right)
$$


where the effective field

$$
-\vec{\nabla} \mathbf{r} H=J_{A F} \sum_{\mathbf{r}^{\prime}} \mathbf{S}_{\mathbf{r}^{\prime}}+\frac{J_{D E}}{2} \sum_{\mathbf{r}^{\prime}} \frac{\mathbf{S}_{\mathbf{r}^{\prime}}}{\sqrt{1+\mathbf{S}_{\mathbf{r}} \cdot \mathbf{S}_{\mathbf{r}^{\prime}}}}
$$

and the sums are over $\mathbf{r}^{\prime}$ nearest-neighbor to $\mathbf{r}$. These equations were solved numerically using a method based on second-order Suzuki-Trotter decomposition of exponential operators [8], to a maximum time of $t_{\max }=$ $440 / J_{D E}$.

The dynamic structure factor $S(\mathbf{q}, \omega)$ for momentum transfer $\mathbf{q}$ and frequency transfer $\omega$, observable in neutron scattering experiments, is given by

$S^{k}(\mathbf{q}, \omega)=\sum_{\mathbf{r}, \mathbf{r}^{\prime}} \exp \left[i \mathbf{q} \cdot\left(\mathbf{r}-\mathbf{r}^{\prime}\right)\right] \int_{-\infty}^{+\infty} \exp (i \omega t) C^{k}\left(\mathbf{r}-\mathbf{r}^{\prime}, t\right) \frac{d t}{\sqrt{2 \pi}}$,

where $C^{k}\left(\mathbf{r}-\mathbf{r}^{\prime}, t\right)$ is the space-displaced, time-displaced spin-spin correlation function defined, with $k=x, y$, or $z$, as $C^{k}\left(\mathbf{r}-\mathbf{r}^{\prime}, t\right)=\left\langle S_{\mathbf{r}}{ }^{k}(t) S_{\mathbf{r}^{\prime}}{ }^{k}(0)\right\rangle-\left\langle S_{\mathbf{r}}{ }^{k}(t)\right\rangle\left\langle{S_{\mathbf{r}^{\prime}}}^{k}(0)\right\rangle$. The displacement $\mathbf{r}$ is in units of the lattice unit cell length $a$. More details on the spin-dynamics methods are given in Refs. 9, 10].

\section{RESULTS}

Our preliminary results show that $\left|\mathbf{m}_{A}\right|=\left|\mathbf{m}_{B}\right|$ at all temperatures for $\left|J_{A F}\right| / J_{D E}<1 / \sqrt{8}$, decreasing smoothly from $\left|\mathbf{m}_{A}\right|=\left|\mathbf{m}_{B}\right|=1$ at low $T$, towards zero as $T$ increases. For $\left|J_{A F}\right| / J_{D E}>1 / \sqrt{8}$ we find that at low temperatures $\left|\mathbf{m}_{A}\right| \neq\left|\mathbf{m}_{B}\right|$, as illustrated in Fig. 1 for $\left|J_{A F}\right| / J_{D E}=0.9$. For this range of interaction strengths our results indicate that $\left|\mathbf{m}_{A}\right|=$ $\left|\left(J_{D E}\right)^{2} /\left(4\left|J_{A F}\right|^{2}\right)-1\right|$ and $\left|\mathbf{m}_{B}\right|=1$ at $T=0$, and Eq.(2) then implies that $\theta=0$ for $\left|J_{A F}\right| / J_{D E}<1 / 2$, $\theta=\pi$ for $\left|J_{A F}\right| / J_{D E}>1 / 2$, and the sublattice magnetizations reduce to $\left|\mathbf{m}_{A}\right|=0$ and $\left|\mathbf{m}_{B}\right|=1$ for $\left|J_{A F}\right| / J_{D E}=1 / 2$. The phase-transition temperatures are obtained from analyses of the sublattice magnetizations for $L=24$, hence there are some uncertainties in these estimates due to finite-size effects.

The phase diagram that we obtained from the analysis of the sublattice magnetizations, shown in Fig. 2, has five different regions: (i) ferromagnetic (F) for low $T$ and small values of $\left|J_{A F}\right| / J_{D E}$; (ii) antiferromagnetic (AF) for moderate $T$ and high values of $\left|J_{A F}\right| / J_{D E}$; (iii) paramagnetic $(\mathrm{PM})$ at high $T$; and two low temperature phases, which we label as (iv) region I for $1 / \sqrt{8}<$ $\left|J_{A F}\right| / J_{D E}<1 / 2$, and (v) region II for $\left|J_{A F}\right| / J_{D E}>$ $1 / 2$. Regions (i)-(iii) are characterized by $\left|\mathbf{m}_{A}\right|=\left|\mathbf{m}_{B}\right|$, with $\mathbf{m}_{A}=\mathbf{m}_{B}$ in the ferromagnetic phase and $\mathbf{m}_{A}=$ $-\mathbf{m}_{B}$ in the antiferromagnetic phase. In contrast, in regions I and II we find that $\left|\mathbf{m}_{A}\right| \neq\left|\mathbf{m}_{B}\right|$ and although our simulations suggest that $\theta=0$ and $\pi$ in regions I and II, respectively, we cannot rule out possible small deviations from the aligned and anti-aligned sublattice spin configurations in the respective regions. The question marks

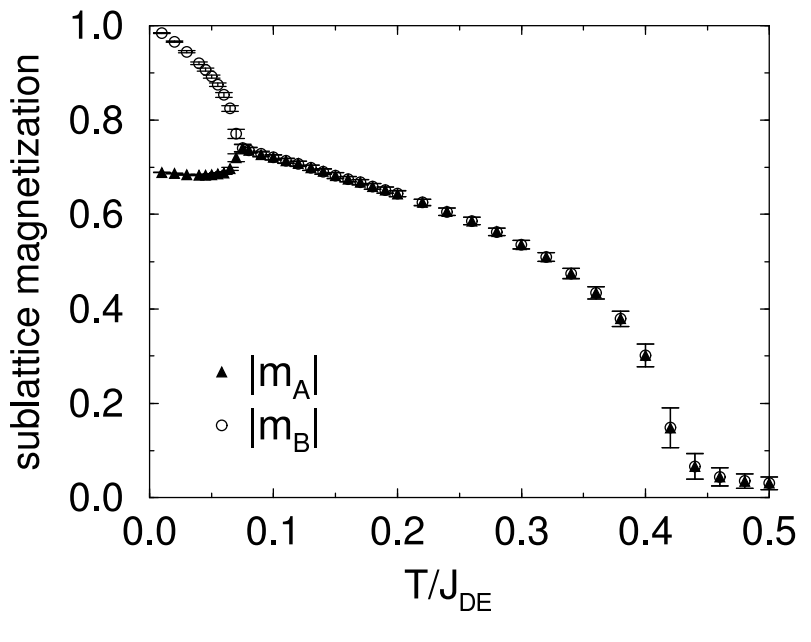

FIG. 1: Sublattice magnetization for $\left|J_{A F}\right| / J_{D E}=0.9$, and lattice size $L=24$.

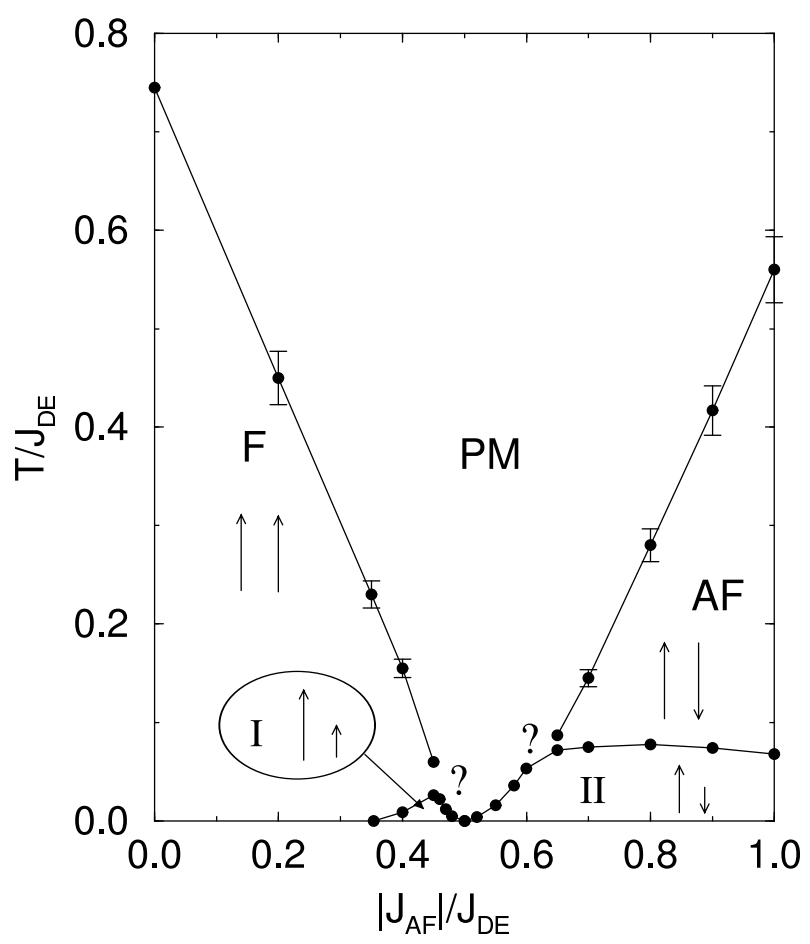

FIG. 2: Phase diagram. When not shown, the error bars are smaller than or of the size of the points and the solid lines simply join the data, guiding the eyes.

(?) in the phase diagram (see Fig. 2) indicate that the points at which the F-PM and AF-PM transition lines join other phase boundaries have not been determined accurately yet. For the pure double-exchange ferromagnetic model $\left(J_{A F}=0\right)$, the critical temperature (F-PM transition point) is $T_{c}{ }^{D E}=0.74515(7) J_{D E}[\underline{6}]$, whereas for the pure Heisenberg model $T_{c}^{S E}=1.442929(77)\left|J_{A F}\right|$ [5] for either ferromagnetic or antiferromagnetic interaction.

Fig. 3 shows a comparison of the dispersion relations 


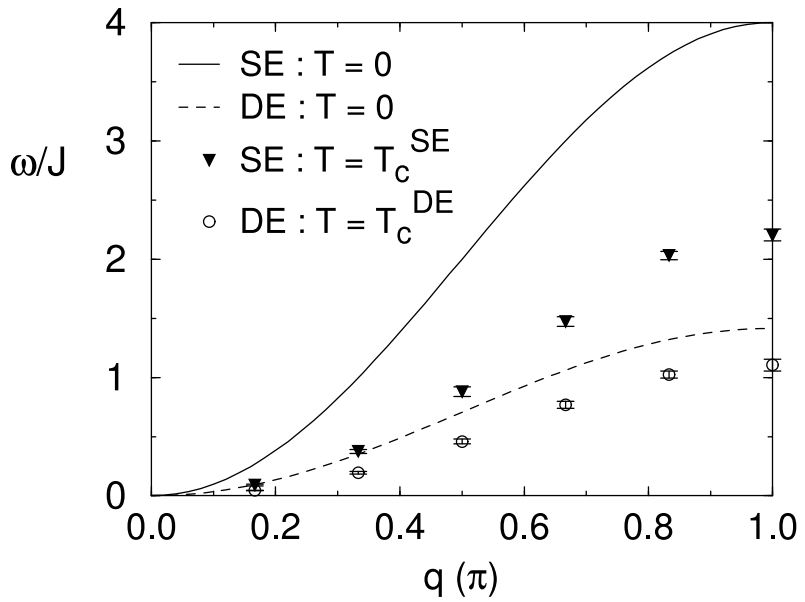

FIG. 3: Dispersion relations for the Heisenberg ferromagnet (pure superexchange model, labeled as SE) and the pure ferromagnetic double-exchange model (DE).

for the pure Heisenberg ferromagnet and for the pure double-exchange ferromagnetic model. The results at $T=0$ correspond to the linear spin-wave theory, and data at the respective critical temperatures are from our simulations.

\section{CONCLUSIONS}

We studied a system of classical spins coupled via competing antiferromagnetic superexchange and ferromagnetic double-exchange interactions. As expected, when the former is much weaker than the latter, the system is ferromagnetic and in the reverse case the system is antiferromagnetic. In addition, we found two other low temperature phases, in which the sublattice magnetizations differ in magnitude and are close to being aligned or anti-aligned, depending on the ratio of the two interaction strenghts. At high temperature the system is paramagnetic. Comparing the dispersion curves we see that spin-waves in the pure double-exchange model occur at lower frequency transfer than in the pure Heisenberg ferromagnet.

\section{Acknowledgments}

This research was supported in part by NSF grant No. DMR-9727714.
[1] See for example: P. Schiffer, A. P. Ramirez, W. Bao, and S.-W. Cheong, Phys. Rev. Lett. 75, 3336 (1995); J. W. Lynn, R. W. Erwin, J. A. Borchers, Q. Huang, A. Santoro, J.-L. Peng, and Z. Y. Li, Phys. Rev. Lett. 76, 4046 (1996); T. G. Perring, G. Aeppli, S. M. Hayden, S. A. Carter, J. P. Remeika, and S.-W. Cheong, Phys. Rev. Lett. 77, 711 (1996); J. W. Lynn, R. W. Erwin, J. A. Borchers, A. Santoro, Q. Huang, J.-L. Peng, and R. L. Greene, J. Appl. Phys. 81, 5488 (1997); L. Vasiliu-Doloc, J. W. Lynn, A. H. Moudden, A. M de Leon-Guevara, and A. Revcolevschi, J. Appl. Phys. 81, 5491 (1997). For a recent review, see for example: J. M. D. Coey, M. Viret, and S. von Molnár, Adv. in Phys. 48, 167 (1999).

[2] C. Zener, Phys. Rev. 82, 403 (1951); P. W. Anderson and H. Hasegawa, Phys. Rev. 100, 675 (1955); P.-G. de Gennes, Phys. Rev. 118, 141 (1960).

[3] See for example: A. J. Millis, R. Mueller, and B. I.
Shraiman, Phys. Rev. B 54, 5405 (1996); and references therein.

[4] E. O. Wollan and W. C. Koehler, Phys. Rev. 100, 545 (1955).

[5] K. Chen, A. M. Ferrenberg, and D. P. Landau, Phys Rev. B 48, 3249 (1993).

[6] A. Caparica, A. Bunker, and D. P. Landau, submitted for publication.

[7] D. P. Arovas and F. Guinea, Phys. Rev. B 58, 9150 (1998).

[8] M. Krech, A. Bunker, and D. P. Landau, Comput. Phys. Comm. 111, 1 (1998); J. Frank, W. Huang, and B. Leimkuhler, J. Comp. Phys. 133, 160 (1997).

[9] K. Chen and D. P. Landau, Phys. Rev. B 49, 3266 (1994).

[10] A. Bunker, K. Chen, and D. P. Landau, Phys. Rev. B 54, 9259 (1996). 